\title{
Clinical and cost-effectiveness analysis of early detection of patients at nutrition risk during their hospital stay through the new screening method CIPA: a study protocol
}

José Pablo Suárez-Llanos ${ }^{*}$, Néstor Benítez-Brito ${ }^{1}$, Laura Vallejo-Torres², Irina Delgado-Brito ${ }^{1}$, Adriá Rosat-Rodrigo ${ }^{3}$, Carolina Hernández-Carballo ${ }^{4}$, Yolanda Ramallo-Fariña ${ }^{2}$, Francisca Pereyra-García-Castro ${ }^{1}$, Juan Carlos-Romero ${ }^{4}$, Nieves Felipe-Pérez ${ }^{4}$, Jennifer García-Niebla ${ }^{3}$, Eduardo Mauricio Calderón-Ledezma ${ }^{4}$,

Teresa de Jesús González-Melián ${ }^{5}$, Ignacio Llorente-Gómez de Segura ${ }^{1}$ and Manuel Ángel Barrera-Gómez ${ }^{3}$

\begin{abstract}
Background: Malnutrition is highly prevalent in hospitalized patients and results in a worsened clinical course as well as an increased length of stay, mortality, and costs. Therefore, simple nutrition screening systems, such as CIPA (control of food intake, protein, anthropometry), may be implemented to facilitate the patient's recovery process. The aim of this study is to evaluate the effectiveness and cost-effectiveness of implementing such screening tool in a tertiary hospital, consistent with the lack of similar, published studies on any hospital nutrition screening system.
\end{abstract}

Methods: The present study is carried out as an open, controlled, randomized study on patients that were admitted to the Internal Medicine and the General and Digestive Surgery ward; the patients were randomized to either a control or an intervention group ( $n=824$, thereof 412 patients in each of the two study arms). The control group underwent usual inpatient clinical care, while the intervention group was evaluated with the CIPA screening tool for early detection of malnutrition and treated accordingly.

CIPA nutrition screening was performed upon hospital admission and classified positive when at least one of the following parameters was met: $72 \mathrm{~h}$ food intake control $<50 \%$, serum albumin $<3 \mathrm{~g} / \mathrm{dL}$, body mass index $<18.5 \mathrm{~kg} / \mathrm{m}^{2}$ (or mid-upper arm circumference $\leq 22.5 \mathrm{~cm}$ ). In this case, the doctor decided on whether or not providing nutrition support.

The following variables will be evaluated: hospital length of stay (primary endpoint), mortality, 3-month readmission, and in-hospital complications. Likewise, the quality of life questionnaires EQ-5D-5 $L$ are being collected for all patients at hospital admission, discharge, and 3 months post-discharge. Analysis of cost-effectiveness will be performed by measuring effectiveness in terms of quality-adjusted life years (QALYS). The cost per patient will be established by identifying health care resource utilization; cost-effectiveness will be determined through the incremental cost-effectiveness ratio (ICER). We will calculate the incremental cost per QALY gained with respect to the intervention.

Discussion: This ongoing trial aims to evaluate the cost-effectiveness of implementing the malnutrition screening tool CIPA in a tertiary hospital.

(Continued on next page)

\footnotetext{
* Correspondence: pablua@hotmail.com

'Endocrinology and Nutrition Department, Hospital Universitario Nuestra Señora de Candelaria, Ctra. Del Rosario 145, Santa Cruz de Tenerife 38010, Spain

Full list of author information is available at the end of the article
} 


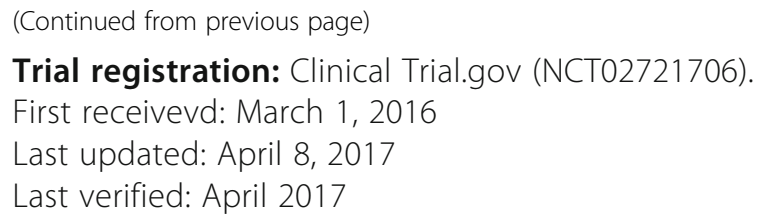

Keywords: Nutrition assessment, Malnutrition, Inpatients, Body composition, Anthropometry, Cost-benefit analysis, Mass screening, Quality of life

\section{Background}

Hospital malnutrition (HM) is a frequent condition in developed countries; its prevalence ranges from 25 to $50 \%$, depending on the study population and the detection method [1-4]. This type of malnutrition is associated with the patient's underlying condition, so that it may be triggered either by the disease-related symptoms (dysphagia, vomiting, diarrhea, malabsorption, etc.), thereby reducing food intake or increasing losses, or by an associated, increased caloric and protein requirement that the patient cannot cover.

Malnutrition in hospitalized patients leads to an exacerbated clinical course, with additionally emerging comorbidities and worsened functional capacity, which in turn lead to a longer hospital stay, increased mortality and health care costs [3-5]. It is, therefore, essential to detect these patients using nutrition screening techniques at hospital admission and thus try to improve their prognosis through nutrition therapy.

HM is as frequent as underrated, so that only few European countries and hospitals apply nutrition screening on admission. In the Spanish multicenter study PREDYCES, where screening was performed using Nutritional Risk Screening 2002 (NRS 2002), 23\% of the hospitalized patients were found to be malnourished [6]. In addition, that malnourished subgroup underwent an aggravated clinical course during their hospital stay [2]. Based on these results, nutrition screening pilot projects are now being implemented in different hospitals throughout the country.

There is no gold standard for hospital nutrition screening, despite the high number of existing screening tools. It is, therefore, best to incorporate one that can be applied according to the hospital characteristics and that primarily identifies patients with the poorest clinical prognosis. In the University Hospital Nuestra Señora de Candelaria (HUNSC), Santa Cruz de Tenerife, Spain, the nutrition screening method CIPA (control of intakes, proteins, anthropometry) was designed by employing tools commonly used in clinical practice.

When CIPA screening was compared with Subjective Global Assessment (SGA) [7, 8] - the standard method for validation, nonetheless not a gold standard - in patients with a non-surgical pathology, those who resulted positive by CIPA screening had a longer mean stay than patients with a negative result (19.53 vs 12.63 days; $p<0.001$ ); this data was not detected by SGA screening. On the other hand, patients identified as positive by either method comprised more cases of death both in hospital ( $7.6 \%$ vs $1.4 \%, p=0.026$; CIPA screening) as well as by the first month post-discharge $(12.7 \%$ vs $3.5 \%, p=0.012)$ [9]. Therefore, in this group of patients, the CIPA screening tool detected those with a comparably worsened clinical course more efficiently than the SGA method.

Although malnourished patients have a worse prognosis and generate higher costs than the rest, the costeffectiveness of performing a hospital nutrition screening is not sufficiently studied. Only few studies have performed an economic evaluation of a malnutrition screening in hospitalized patients. Kruzienga HM et al. analyzed the implementation of the screening method SNAQ (Short Nutritional Assessment Questionnaire) and concluded that its implementation was cost effective, mainly in the fragile patient [10]. The NICE guidelines on Nutrition Support in Adults [11] indicated that screening is likely to be cost effective but highlighted a high degree of uncertainty due to "the weakness in the methodologies and designs of identified studies."

This study aims to shed light onto this area and study the clinical and economic impact through a randomized, controlled trial on the implementation of a nutrition screening method, in this particular case the CIPA method, in a tertiary hospital.

\section{Methods}

\section{Trial design}

This study is a two-armed, randomized, controlled trial, comprising an intervention arm, where patients were screened for malnutrition using CIPA at hospitalization, and a control arm, where patients were not screened but diagnosed according to standard clinical practice.

\section{Subjects}

Patient inclusion criteria:

1. Patient admitted to the hospitalization ward of Internal Medicine or General and Digestive Surgery of the HUNSC. 
2. Formal consent to participate in the study.

3. Age of 18 or more years.

Patient exclusion criteria:

1. Patient treated with nutrition support before finishing the CIPA screening procedure.

2. Patient transferred from other ward.

3. Patient with an expected length of stay of less than $72 \mathrm{~h}$.

4. Unfeasible CIPA screening for whatever reason.

5. Patient with poor short-term prognosis.

6. Bed assignment at hospital admission nonrandomized.

7. Patient participating in other research study.

8. Pregnancy.

\section{Setting and recruitment}

Patients were recruited in the hospitalization area of the HUNSC, in the wards of Internal Medicine and General and Digestive Surgery. These departments were chosen for integrating the patients with the highest clinical variability and comorbidity. Recruitment was conducted in two phases: the first, at the time of hospitalization, in which the patient was confirmed to meet the inclusion or exclusion criteria and asked to sign the informed consent, thereby becoming potentially eligible. The second phase took place at $72 \mathrm{~h}$, when the result of the CIPA nutrition screening was obtained, point at which the patient was verified not to have received nutrition treatment by that time and eventually became part of the sample. Otherwise, the patient was excluded from the study according to criterion 1 ).

\section{Random assignment}

Both scheduled and emergency admission patients were allocated by the department of hospital admission to one of the participating wards, following the randomization plan, which was generated by simple random sampling and delivered by the data manager of the project. This randomization facilitated trial execution and prevented protocol errors, as the group to which each patient belonged was defined by its location.

Both the Internal Medicine and the General and Digestive Surgery ward are divided into two hospitalization wings. At the time of the study, due to the progressive introduction of the nutrition screening in the HUNSC, one of the two wings of either ward conducted the CIPA screening at hospital admission (intervention wards), while the other did not (control wards, where standard practice was performed).

\section{Blinding}

Due to the nature of the intervention, neither enrolled patients nor the clinical research team could have been blinded to the group they belonged to, although patients were not informed until they signed the consent of their group. The doctors responsible for treatment did not know whether or not patients were enrolled in the study for not to create an intervention bias on the patient. Data analysis is going to be blinded to the intervention assignment.

\section{Interventions}

Patients in the intervention group were subjected to CIPA nutrition screening, which resulted positive if one of the following conditions was met: (1) $72 \mathrm{~h}$ food intake control below 50\% [12]; (2) serum albumin < $3 \mathrm{~g} / \mathrm{dL}$; (3) body mass index $(\mathrm{BMI})<18.5 \mathrm{~kg} / \mathrm{m}^{2}$ or mid-upper arm circumference $(M U A C) \leq 22.5 \mathrm{~cm}$ [13], the latter when the patient could not be weighed and measured. The flow chart in Fig. 1 reflects the health care personnel's staff's tasks to accomplish the screening process.

When patients were admitted through the emergency room or on a scheduled basis for any medical reason, the nutrition screening process started on ward admission. Conversely, if patients were admitted for scheduled surgery, nutrition screening began thereafter, when they arrived at the ward.

In case of a positive screening result, the attending doctor would evaluate the adequacy of a nutrition treatment. To this end, if the patient was unable to feed orally, the doctor requested a referral to the Endocrinology and Nutrition Service, whose doctors and nurses were in charge of a nutrition assessment and the administration of the artificial nutrition they considered appropriate. In the case the patient was able to eat by mouth, a dietitian would adjust the diet and monitor the patient's progress on the third and tenth day, if necessary. In addition, according to the internal protocol, the patient received two specific oral nutrition supplements (ONS) per day, depending on their comorbidities.

As for the control group, routine clinical practice without any nutrition screening was carried out, so that the responsible doctor may have requested the nutrition parameters and considered specific nutrition treatment if appropriate.

Regardless of the group they belong to, patients with nutrition support during hospitalization may have continued that prescribed support for at least three more months past discharge, provided that the responsible doctor considered the measure appropriate and their pathology was included in the financing list of such treatments of the Spanish National Health System (SNHS). Figure 2 shows the intervention algorithm.

\section{Ethics}

The Scientific and Ethics Committees of the HUNSC approved the study protocol. The study is being performed 

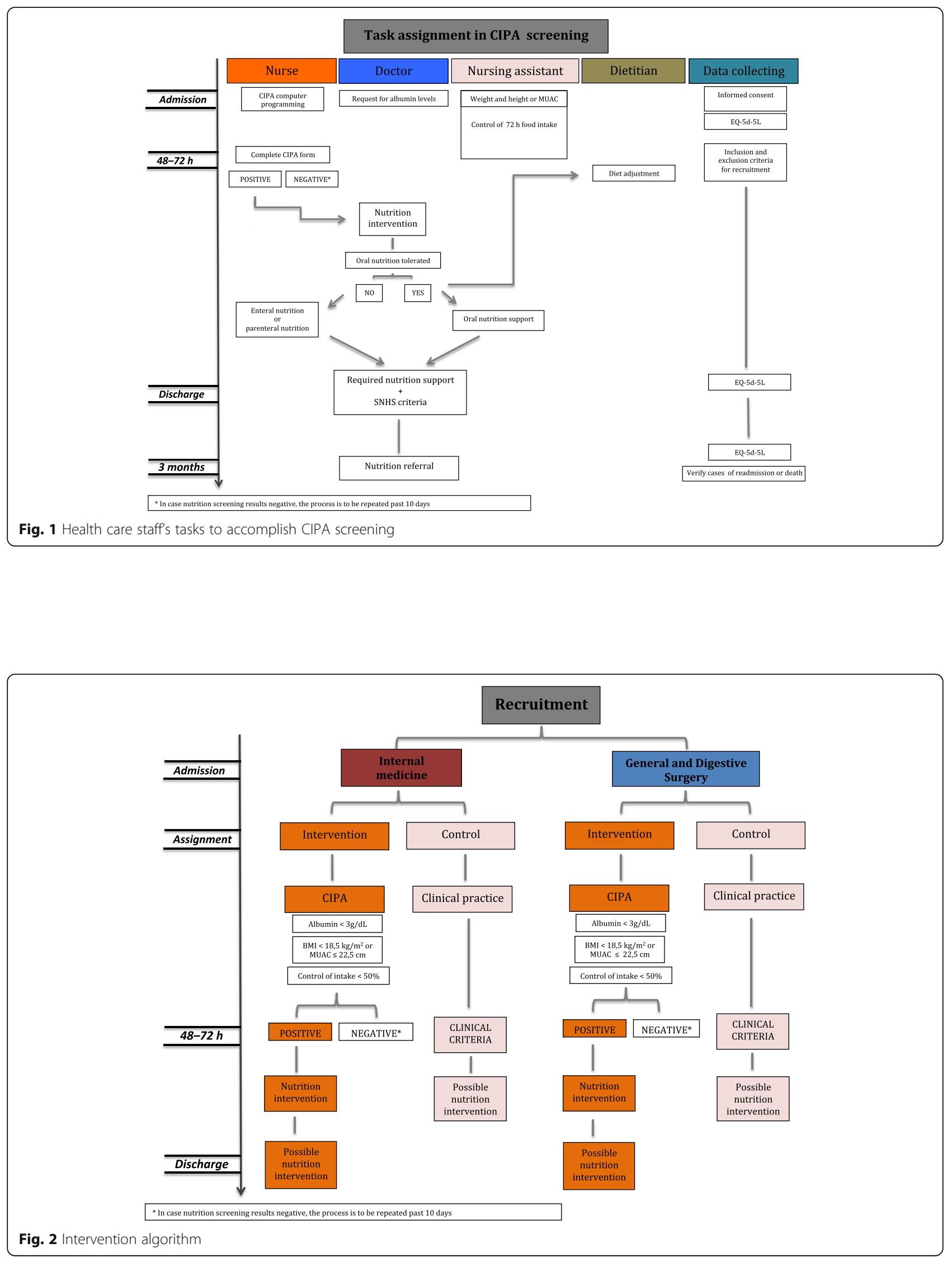
in accordance with Good Clinical Practice Standards, applicable local regulatory requirements, and the recommendations of the Declaration of Helsinki.

\section{Endpoints \\ Primary endpoint}

The primary endpoint of this study is the difference in mean length of hospital stay between the two compared groups. A difference of 2.6 days is considered clinically significant [13].

\section{Secondary endpoints}

\section{Mortality and morbidity}

1. Rate of mortality in in-hospital patients.

2. Rate of mortality in the course of three months postdischarge.

3. Comorbidity will be assessed using the Charlson Index (CI) [14], which comprises 19 categories of the International Classification of Diseases, (10th revision) diagnose codes (ICD-10). It is based on a set of risk factors for the 1-year mortality risk and can be applied to establish the association between the life expectancy of a patient and the resources a health care system must allocate.

\section{Complications}

1. Readmission rates, i.e., within 3 months post-discharge.

2. Rate of surgical interventions during hospital stay.

3. Incidence of clinical in-hospital complications according to the Classification of Hospital-Acquired Diagnoses (CHADx) [15]. The CHADx tool is a hierarchical classification of encoded diagnoses based on the International Classification of Diseases-10 (ICD-10), which allows quantification of the burden of in-hospital complications.

4. Surgical complications according to the ClavienDindo classification, a standardized and internationally validated scoring system. The system allows for the severity of the complication and its interference in the clinical course of the operated patients. The highest grade complication (grades I-V) experienced by each patient was recorded [16].

\section{Treatment}

1. Rate of prescribed nutrition treatments.

2. Period prior to nutrition treatment.

3. Characteristics of the nutrition treatment (duration, type, indications, prolongation following discharge, changes in the treatment, number of visits of the dietitian, and number of referrals).

\section{Quality of life}

1. Health-related quality of life (HRQoL) was measured using the EQ-5D-5 L questionnaire. This generic, self-reported description assesses five domains: mobility, self-care, usual activity, pain or discomfort, and anxiety or depression. Each dimension is scored on a scale of 1 to 5 , depending on whether the respondent has no problems, slight, moderate, severe, or extreme problems with each dimension. This descriptive system defines 3125 health states. Each health state can then be converted into a single summary index by applying a formula that associates weights to each state, based on evaluations from general population samples, developed by the EuroQoL group. Upon application of these weights, an EQ-5D index score of one represents full health, a score of zero is equivalent to death, and negative scores represent health states worse than death [17].

\section{Biochemical determinations}

1. Plasma albumin was determined by colorimetry, using a Roche/Hitachi Cobas C 702 Autoanalyzer (Roche Diagnostics). This parameter was requested automatically within $48 \mathrm{~h}$ post-admission. Results are expressed in $\mathrm{g} / \mathrm{dL}$.

\section{Anthropometric determinations}

1. BMI: Weight $(\mathrm{kg})$ and height $(\mathrm{cm})$ were obtained with the patient barefoot, dressed in hospital pajamas, using calibrated Seca 220 scales that include a height rod. In the case of patients confined to bed, where weight and height could not be determined, MUAC $(\mathrm{cm})$ was obtained on the patient's non-dominant arm, at the midpoint between the acromion and olecranon, surrounding the arm with a measuring tape (Seca 201) without pressing the limb. This data was collected by nurses and nursing assistants, when the patient entered the hospital ward, and was then transferred to the electronic medical record (EMR).

Health care utilization Health care utilization includes staff time and material required to implement the malnutrition screening tool CIPA, duration of tube feeding or any other nutritional treatment, visits from other specialists during hospitalization, in-hospital complications, hospital length of stay, and readmissions and other health care contacts within 3 months after discharge.

In addition, the following patient information was collected upon admission: initials, the assigned ward, clinical record, nationality, age, sex, type of admission (emergency or scheduled), date of and diagnosis on admission. Subsequently, the diagnosis-related group (DRG) as well as the severity-based DRG were established. 


\section{Measurement procedures}

Data collection is being managed using an electronic case report form (CRF), designed for this study and complemented with EMR information and patient questionnaires conducted by phone 3 months past discharge.

The project data manager compiles all clinical data from the EMR, except for the EQ-5D-5 L questionnaires, which are being collected at hospital admission, discharge, and 3 months post-discharge, and the DRG and severity-based DRG, which are presented by the Management Unit following hospital discharge. The main data and laboratory parameters of included patients are directly recorded in the CRF. The CI and the CHADx and Clavien-Dindo classifications are documented by the medical staff subsequent to the patient's discharge.

\section{Statistical methods Sample size calculation}

According to the study of Kruizenga et al. [10], 412 patients per arm (a total of 824 patients for this study) are required to detect a difference in hospital stay of 2.6 days, assuming a standard deviation of 8 days in the intervention group and 13.3 days in the control group. Calculations were performed considering a two-tailed power of $90 \%$, an alpha of 0.05 , and $10 \%$ of potential losses.

\section{Statistical analysis}

Descriptive data analysis will present qualitative variables as frequencies and percentages, and continuous variables as means, medians, and standard deviations. Normality shall be verified by the Kolmogorov-Smirnov normality test. In bivariate analysis, depending on the nature of the variables, the parametric hypothesis test Student's $t$-test or the non-parametric Mann-Whitney test will be used. To assess the effects of the intervention, changes in the primary and secondary outcomes will be analyzed by a between-group ANCOVA variance test. The analysis will control for the type, age, and sex of the patient, as well as the responsible medical unit. Patients admitted to Internal Medicine will be categorized into the four groups (1) cardiovascular, (2) neoplastic, (3) infectious, and (4) other patients, whereas patients in the Digestive and General Surgery services will be divided into the categories (1) neoplastic, (2) infectious, (3) obstructive, (4) inflammatory, and (5) others. The economic evaluation and statistical analyses of individual variables will be conducted in accordance with the intention-to-treat principle.

All tests will be two-sided with a type I error of $5 \%$. Statistical analyses will be performed using the Statistical Package for Social Sciences (SPSS v.23, Chicago, IL, USA).

\section{Cost-effectiveness analysis}

We will analyze the cost and cost-effectiveness of implementing the malnutrition screening tool CIPA versus standard practice in patients admitted to the Internal Medicine and General and Digestive Surgery wards. These analyses will be in line with accepted economic evaluation methods.

The cost will be assessed from a National Health Service perspective and, therefore, only include medical costs incurred by the health care service. Based on data from the hospital as well as the national sources, we will assign a unit cost to each component of health care utilization collected in this trial.

The cost-effectiveness measure will be the incremental cost per quality-adjusted life year (QALY) gained. QALYs will be calculated based on the HRQoL and mortality data collected during the trial. Patient-specific utility profiles will be created assuming a straight-line relation between all patients' EQ-5D-5 L scores from either follow-up point. The QALYs experienced by each patient from baseline to 3 months will be calculated as the area underneath this profile. We will investigate differences in baseline characteristics and, if necessary, use regression methods to control for them.

Cost-effectiveness will be summarized as the incremental cost-effectiveness ratio (ICER) by dividing the estimated differences in cost by the differences in QALYs. Non-parametric methods to calculate confidence intervals around the ICER, based on bootstrapped estimates of the mean cost and QALY differences, will be used. Further, the bootstrap replications will be used to create a costeffectiveness acceptability curve, which will reveal the probability that each alternative is cost effective for different values of willingness to pay for an additional QALY. We will also subject the results to extensive, deterministic (one, two-, and multi-way) sensitivity analyses.

\section{Duration of fieldwork}

The recruitment phase lasted for 15 months. Patients are being followed up for three more months post-discharge. Thereafter, a subsequent phase of data cleansing, identification of unit costs, and data analyses will take place.

\section{Monitoring}

Trial monitoring is the responsibility of the research team in charge of all quality control activities, assessing adherence to the trial protocol, timely work plan execution, and comprehensiveness of data acquisition and data quality. The databases have been designed to avoid, for each variable, downloading of inappropriate values.

\section{Trial status}

This study is ongoing, the investigators are still collecting data. 


\section{Discussion}

This research project evaluates the effectiveness and cost-effectiveness of implementing a hospital nutrition screening (in this case the CIPA method) in a detailed manner, comprising a large number of variables to analyze the effect of its introduction from different perspectives. On the one hand, clinical variables, such as medical complications during the admission process, mean length of stay, mortality, readmission, or even patient-directed procedures are assessed. On the other hand, data collection includes costs of the visits from dietitians and other skilled health personnel, as well as the costs of nutrition support, not only ONS, but also tube feeding or parenteral feeding, where necessary.

Disease-associated malnutrition is a perfect target for screening due to its high prevalence and the established therapeutic measures that can mitigate its deleterious effects when applied timely, thereby allowing clinical and economic improvement [18-22]. However, there is no ideal method of nutrition screening, as those that best predict the patient's clinical course are the most complex to perform and require substantial efforts for implementation in hospitals with many patients, as e.g., SGA [7] or NRS2002 [6], while others that are easier and more feasible to carry out, do not predict that course so well. Consequently, the ideal screening system should adapt to the hospital characteristics, predict patient outcome, be workable by most caregivers, and should be inexpensive and not time-consuming [23-25].

The CIPA screening meets all these criteria: (1) it employs tools used in everyday clinical practice that do not require specialized personnel; (2) it is quick to perform; (3) it is objective; (4) it is inexpensive, since the only extra cost is the general determination of serum albumin at hospital admission, which is negligible for a measure with foreseeable, significant savings; (5) it is able to predict the patients' prognosis, as in a study in 221 non-surgical patients the CIPA screening detected patients with a comparably longer mean length of stay of one week and a higher mortality, similar to the findings of another early study that allowed its development $[13,14]$.

Ethical constraints on prospective studies of nutrition intervention in malnourished patients resulted in scientific evidence of the benefit of treating these patients primarily from retrospective studies. The outstanding work of Phillipson TJ et al. [20] revealed that treatment with ONS decreased the mean length of stay, readmission rate, and health cost. Likewise, two meta-analyses, recently published by Elia $\mathrm{M}$ et al., describe the clinical and economic benefits of ONS treatment in malnourished, in-hospital patients and community settings $[21,22]$. Furthermore, a recently published, first prospective, randomized, double-blind study showed that a nutrition supplement decreases the incidence of mortality in in-hospital patients during their stay and up to 3 months post-discharge [23].

Using the SNAQ tool, a study by Kruizenga HM et al. [10] analyzed the cost-effectiveness of the implementation of a nutrition screening at hospital admission. The authors mainly evaluated the decrease in the mean stay as a measure to reduce costs, while taking into account extra expenses due to ONS treatment and dietitian visits, finding the tool to be cost effective primarily in the fragile patient.

Although a poor nutrition status relates to a limited HRQoL both in inpatients as well as non-institutionalized patients, very few studies have assessed HRQoL associated with the nutrition status or treatment in risk patients [26-28], most of them focusing on clinical parameters. The present study includes the analysis of the state of HRQoL by means of the EQ-5D-5 L questionnaire, not only as a generic reference in terms of the association of the nutrition status with the HRQoL, but also because QALYs are the most widely used measure to evaluate the cost-effectiveness of an intervention in the field of health care [29].

The limitations of the present study include the impossibility to perform a double-blind study, as the responsible doctor should know if the patient has screened positive or negative to act on their clinical criterion. In some instances, standard clinical practice may also cause delays in adapting the pattern of nutrition treatment to the screening result, and treatment might be overlooked at hospital discharge even when required by the patient.

It is worth noting that the SNHS only covers the cost of post-discharge nutrition supplements under very specific conditions, e.g., in cancer patients, patients with dysphagia, neurodegenerative diseases, and similar. Consequently, many of the patients who need nutrition support in hospital would not be able to continue after their discharge. For this reason, the number of patients with continued nutrition supplementation after discharge is expected to be rather low in this study.

At the same time, due to training and greater awareness about diagnosis and treatment of malnutrition among the clinical staff of the HUNSC, particularly in the surgical wards, the number of patients excluded for having nutritional support before finalizing the CIPA screening has been higher than expected, which led to an extension of the recruitment period.

Therefore, with the scientific rationale in favor of a nutrition management of malnourished patients and with tools for early detection of this condition at hand, it is foreseeable that the implementation of a cheap and simple hospital nutrition screening results in a cost-effective intervention. However, properly designed and methodologically unbiased studies are required to evaluate this relationship. Given the scarcity of literature in regard to 
this type of evaluations, we hope to fill the evidence gap between hospital nutrition screening and health care cost savings with this study, and, if proven cost effective, promote the universal implementation of the CIPA screening system in hospitals through appropriate political strategies.

\section{Abbreviations}

BMI: Body mass index; CHADx: Classification of hospital-acquired diagnoses; Cl: Charlson index; CIPA: Control of food intake, protein and anthropometry; CRF: Case report form; DRG: Diagnosis-related group; EMR: Electronic medical record; HM: Hospital malnutrition; HRQoL: Health-related quality of life; HUNSC: Hospital Universitario Nuestra Señora de Candelaria; ICD-10: International classification of diseases (10th revision); ICER: Incremental cost-effectiveness ratio; MUAC: Mid-upper arm circumference; NRS2002: Nutritional risk screening 2002; ONS: Oral Nutrition supplements; QALYs: Quality-adjusted life years; SGA: Subjective global assessment; SNAQ: Short nutritional assessment questionnaire; SNHS: Spanish national health system

\section{Acknowledgements}

The authors would like to thank the Servicio de Evaluación de Tecnologías Sanitarias del Servicio Canario de la Salud (SESCS) for collaborating on design and supervision of the project.

Ma Ángeles Tirado-Trujillos.

Health Management Service.

Hospital Universitario Nuestra Señora de Candelaria, Tenerife, España. Juan Antonio Pérez-Pérez.

Computer Science Department.

Hospital Universitario Nuestra Señora de Candelaria, Tenerife, España.

Carmen Muñoz-Darias.

Health Management Service.

Hospital Universitario Nuestra Señora de Candelaria, Tenerife, España.

Cristina Valcárcel-Nazco.

Canary Foundation for Health Research (FUNCANIS) Evaluation Service of the Canary Health System (SESCS), Research Network on Health Services Chronic Disease (REDISSEC), Canary Center for Biomedical Research (CIBICAN).

Déborah Alonso-Modino.

Neurology Department.

Hospital Universitario Nuestra Señora de Candelaria, Tenerife, España.

José Enrique Palacio-Abizanda.

Endocrinology and Nutrition Department.

Hospital Universitario Nuestra Señora de Candelaria, Santa Cruz de Tenerife, Spain.

Lina Pérez-Méndez.

Research Unit.

Hospital Universitario Nuestra Señora de Candelaria and Primary Healthcare, Santa Cruz de Tenerife, Spain.

CIBER CIBER, Respiratory diseases.

Instituto de Salud Carlos III, Madrid, Spain.

Nayra Pérez-Delgado.

Biochemistry and laboratory DEpartment.

Hospital Universitario Nuestra Señora de Candelaria, Tenerife, España.

María Alicia López-Frías.

Geriatrics Department.

Hospital Universitario Nuestra Señora de Candelaria, Tenerife, España.

Maria Araceli García-Núñez.

Endocrinology and Nutrition Department.

Hospital Universitario Nuestra Señora de Candelaria, Santa Cruz de Tenerife, Spain.

José Gregorio Oliva-García.

Endocrinology and Nutrition Department.

Hospital Universitario Nuestra Señora de Candelaria, Santa Cruz de Tenerife, Spain.

Mercedes Muro-de Fuentes.

Biochemistry and laboratory DEpartment.

Hospital Universitario Nuestra Señora de Candelaria, Tenerife, España.

Pedro Serrano-Aguilar.

Canary Foundation for Health Research (FUNCANIS) Evaluation Service of the Canary Health System (SESCS), Research Network on Health Services Chronic Disease (REDISSEC), Canary Center for Biomedical Research (CIBICAN).
Network Health Technology Assessment and Performance of National Health System (RedETS).

Ruth Suárez-Darias.

General and digestive surgery Department

Hospital Universitario Nuestra Señora de Candelaria, Tenerife, España.

Delia Galindo-Mireles.

Health Management Service.

Hospital Universitario Nuestra Señora de Candelaria, Tenerife, España.

Lidia García-Pérez.

Canary Foundation for Health Research (FUNCANIS) Evaluation Service of the Canary Health System (SESCS), Research Network on Health Services Chronic

Disease (REDISSEC), Canary Center for Biomedical Research (CIBICAN).

Network Health Technology Assessment and Performance of National Health System (RedETS).

Carlos Damián González-Díaz.

General and digestive surgery Department.

Hospital Universitario Nuestra Señora de Candelaria, Tenerife, España.

Lorena González Hernández.

General and digestive surgery Department

Hospital Universitario Nuestra Señora de Candelaria, Tenerife, España.

\section{Availability of data and materials}

No data have been generated from this study protocol. Data collection tools are available from the authors upon request.

\section{Funding}

The study is financially supported by the Instituto de Salud Carlos III (ISCIII), Subdirección General de Evaluación y Fomento de la Investigación, Plan Estatal de Investigación Científica y Técnica y de Innovación 2013-2016, and the Fondo Europeo de Desarrollo Regional_FEDER_-(PI14/01226).

The funders had no role in study design, data collection and analysis, decision to publish, or preparation of the manuscript.

\section{Authors' contributions}

JPSL is the principle investigator and conceptualized and designed the CIPA study together with the following investigators: NBB, LVT, IDB, ARR, CHC, FPGC, JCRP, NFP, JGN, YRF, and ILGS. JPSL and NBB were in charge of project management and supervision and coordinated the different investigators. JPSL, NBB, YRF, and IDB wrote the first draft of this publication with contributions from ARR, CHC, FPGC, JCRP, NFP, JGN, EMCL, and ILGS (the "interventions" section), and LVT and YRF (the "cost-effectiveness analysis" section). ARR, CHC, NFP, JGN, EMCL, and IDB took over patient data and informed consent collecting and transferred the data into the data base. IDB was in charge of collecting the life quality questionnaires from the patients. NBB and FPGC supervised proper internal functioning of the CIPA nutrition screening. TJGM collaborated in coordinating nurses and nursing assistants, All authors contributed to the revisions and read and approved the final manuscript.

\section{Competing interests}

The authors declare that they have no competing interests.

\section{Consent for publication}

Not applicable.

\section{Ethics approval and consent to participate}

The ethics committee of University Hospital Nuestra Señora de Candelaria gave it's approval for the carrying out of this study on the date 6 February 2015 (project number PI14/01226).

All patients included in the study have signed informed consent.

\section{Publisher's Note}

Springer Nature remains neutral with regard to jurisdictional claims in published maps and institutional affiliations.

\section{Author details}

'Endocrinology and Nutrition Department, Hospital Universitario Nuestra Señora de Candelaria, Ctra. Del Rosario 145, Santa Cruz de Tenerife 38010, Spain. ${ }^{2}$ Canary Foundation for Health Research (FUNCANIS) Evaluation Service of the Canary Health System (SESCS), Research Network on Health Services Chronic Disease (REDISSEC), Canary Center for Biomedical Research 
(CIBICAN), Santa Cruz de Tenerife, Spain. ${ }^{3}$ General and digestive surgery Department, Hospital Universitario Nuestra Señora de Candelaria, Santa Cruz de Tenerife, Spain. ${ }^{4}$ Internal Medicine Department, Hospital Universitario Nuestra Señora de Candelaria, Santa Cruz de Tenerife, Spain. ${ }^{5}$ Hospital Universitario Nuestra Señora de Candelaria, Santa Cruz de Tenerife, Spain.

Received: 24 December 2016 Accepted: 1 April 2017

Published online: 20 April 2017

\section{References}

1. Edington J, Boorman J, Durrant ER, Perkins A, Giffin CV, James R, et al. Prevalence of malnutrition on admission to four hospitals in England. The malnutrition prevalence group. Clin Nutr. 2000;19:191-5.

2. Alvarez-Hernández J, Planas Vila M, León-Sanz M, Garcíade Lorenzo A, Celaya-Pérez S, García-Lorda P, et al. Prevalence and costs of malnutrition in hospitalized patients; the PREDyCES Study. Nutr Hosp. 2012;27:1049-59.

3. Sorensen J, Kondrup J, Prokopowicz J, Schiesser M, Krahenbuhl L, Meier R, et al. EuroOOPS: an international, multicentre study to implement nutritional risk screening and evaluate clinical outcome. Clin Nutr. 2008;27:340-9.

4. Correia Ml, Campos AC. Prevalence of hospital malnutrition in Latin America: the multicenter ELAN study. Nutrition. 2003;19:823-5.

5. Correia MI, Waitzberg DL. The impact of malnutrition on morbidity, mortality, length of hospital stay and costs evaluated through a multivariate model analysis. Clin Nutr. 2003;22:235-9.

6. Kondrup J, Rasmussen HH, Hamberg O, Stanga Z. Nutritional risk screening (NRS 2002): a new method based on an analysis of controlled clinical trials. Clin Nutr. 2003;22:321-36.

7. Detsky AS, McLaughlin JR, Baker JP, Johnston N, Whittaker S, Mendelson RA, et al. What is subjective global assessment of nutritional status? J Parenter Enteral Nutr. 1987;11:8-13.

8. Van Bokhorst-de van der Schueren MA, Guaitoli PR, Jansma EP, de Vet HC. Nutrition screening tools: does one size fit all? A systematic review of screening tools for the hospital setting. Clin Nutr. 2014;33:39-58.

9. Suárez Llanos JP, Mora Mendoza A, Benítez Brito N, Delgado Brito I, Pérez Méndez L, Pereyra-García Castro F, et al. Validity of the new nutrition screening tool CIPA in non-surgical inpatients. Arch Med Sci. 2017. (in press).

10. Kruizenga HM, Seidell JC, de Vet HC, Wierdsma NJ, van Bokhorst-de van der Schueren MA. Development and validation of a hospital screening tool for malnutrition: the short nutritional assessment questionnaire (SNAQ). Clin Nutr. 2005:24:75-82.

11. NICE. Nutrition support in adults Oral nutrition support, enteral tube feeding and parenteral nutrition. Clinical guideline [CG32]. London: National Collaborating Centre for Acute Care; 2006.

12. Benítez BN, Suárez Llanos JP, Fuentes FM, Oliva García JG, Delgado Bl, et al. Relationship between mid-upper arm circumference and body mass index in inpatients. Plos One. 2016;11(8):1-10.

13. Suárez Llanos JP, Benítez BN, Oliva García JG, Pereyra-García Castro F, López Frias MA, García Hernández A, et al. Introducing a mixed nutritional screening tool (CIPA) in a tertiary hospital. Nutr Hosp. 2014;29:1149-53.

14. Gil-Bona K, Sabaté A, Miguelena Bovadilla JM, Adroer R, Koo M, Jaurrieta E. Charlson index and the surgical risk scale in the analysis of surgical mortality. Cir Esp. 2010;88(3):174-9.

15. Trentino KM, Swain SG, Burrows SA, Sprivulis PC, Daly FF. Measuring the incidence of hospital acquired complications and their effect on length of stay using CHADx. Med J Aust. 2013;1998(8):543-7.

16. Dindo D, Demartines N, Clavien PA. Classification of surgical complications: a new proposal with evaluation in a cohort of 6336 patients and results of a survey. Ann Surg. 2004;240(2):205-13.

17. Herdman M, Guidex C, Lloyd A, Janssen M, Kind P, Parkin D, et al. Development and preliminary testing of the new five-level version of EQ-5D (EQ-5D-5 L). Qual Life Res. 2011;20:1727-36.

18. Stratton $\mathrm{R}$, Hackston A, Longmore D, Dixon R, Price $\mathrm{S}$, et al. Malnutrition in hospital outpatients and inpatients: prevalence, concurrent validity and ease of use of the "malnutrition universal screening tool" ("MUST") for adults. Br J Nutr. 2004;92:199-808

19. Benítez Brito N, Mora Mendoza A, Suárez Llanos JP, Delgado Brito I, Pérez Méndez LI, Herrera Rodríguez EM, et al. Concordance in the results of control intake performance of $72 \mathrm{~h}$ by different health professionals in a tertiary hospital. Nutr Hosp. 2015;32(6):2895-9.
20. Philipson TJ, Snider JT, Lakdawalla DN, Stryckman B, Goldman DP. Impact of oral nutritional supplementation on hospital outcomes. Am J Manag Care. 2013;19:121-8

21. Elia M, Normand C, Norman $K$, Laviano A. A systematic review of the cost and cost effectiveness of using standard oral nutritional supplements in the hospital setting. Clin Nutr. 2016;35(2):370-80.

22. Elia M, Normand C, Laviano A, Norman K. A systematic review of the cost and cost effectiveness of using standard oral nutritional supplements in community and care home settings. Clin Nutr. 2016;35(1):125-37.

23. Deutz EN, Matheson EM, Matarese LE, Luo M, Baggs GE, Nelson JN, Hegaz RA, et al. Readmission and mortality in malnourished, older, hospitalized adults treated with a specialized oral nutritional supplement: a randomized clinical trial. Clin Nutr. 2016:35:18-26.

24. Ferguson M, Capra S, Bauer J, Banks M. Development of a valid and reliable malnutrition screening tool for adult acute hospital patients. Nutrition. 1999;15:458-64.

25. Waitzberg DL, Correia MI. Nutritional assessment in the hospitalized patient. Curr Opin Clin Nutr Metab Care. 2003;6:531-8.

26. Burden ST, Brierley ER. Evaluation of adherence to a nutrition screening programme over a 5-year period. Eur J Clin Nutr. 2014;68:847-52.

27. Rasheed S, Woods RT. Malnutrition and quality of life in older people: a systematic review and meta-analysis. Ageing Res Rev. 2013:12:561-6.

28. Rasheed S, Woods RT. An investigation into the association between nutritional status and quality of life in older people admitted to hospital. J Hum Nutr Diet. 2014;27:142-51.

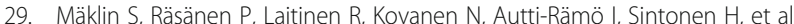
Quality-adjusted life-years for the estimation of effectiveness of screening programs: a systematic literature review. Int J Technol Assess Health Care. 2012:28:145-51.

\section{Submit your next manuscript to BioMed Central and we will help you at every step:}

- We accept pre-submission inquiries

- Our selector tool helps you to find the most relevant journal

- We provide round the clock customer support

- Convenient online submission

- Thorough peer review

- Inclusion in PubMed and all major indexing services

- Maximum visibility for your research

Submit your manuscript at www.biomedcentral.com/submit
) Biomed Central 